\title{
Higher-derivative gauge theories from noncommutative geometry
}

\author{
Walter D. van Suijlekom* \\ Institute for Mathematics, Astrophysics and Particle Physics \\ Radboud University Nijmegen \\ Heyendaalseweg 135 \\ 6525 AJ, Nijmegen \\ E-mail: waltervsemath.ru.nl
}

We review some recent results on the renormalizability of the spectral action for the Yang-Mills system on a flat 4-dimensional background manifold, focusing on its asymptotic expansion. Interpreting the latter as a higher-derivative gauge theory, a power-counting argument shows that it is superrenormalizable. We explicitly compute the asymptotic expansion of the spectral action in this case up to order $\Lambda^{-4}$, including for the first time also the heat coefficient $a_{8}$.

Proceedings of the Corfu Summer Institute 2011 School and Workshops on Elementary Particle Physics and Gravity

September 4-18, 2011

Corfu, Greece

\footnotetext{
* Speaker.
} 


\section{Introduction}

The object of study in this paper is the spectral action for the Yang-Mills (YM) system, given by

$$
S[A]=\operatorname{Tr} f\left(D_{A} / \Lambda\right)-\operatorname{Tr} f(D / \Lambda),
$$

with $D_{A}$ the Dirac operator coupled to a $S U(N)$ gauge field $A_{\mu}$. The function $f$ is real and even, and such that $f\left(D_{A} / \Lambda\right)$ is traceclass, and $\Lambda$ is a real cutoff parameter. In comparison to [4, 5], we have subtracted the purely gravitational term $\operatorname{Tr} f(D / \Lambda)$, focusing on the Yang-Mills part.

Already in [4] it was shown that in four dimensions there is an asymptotic expansion as $\Lambda \rightarrow \infty$ for $S[A]$ such that, modulo negative powers of $\Lambda$ the spectral action yields the Yang-Mills action. The remaining terms in this asymptotic expansion (as $\Lambda \rightarrow \infty$ ) turn out to be of interest too, appearing in [6] and my previous work [18, 17]. We write the asymptotic expansion (as $\Lambda \rightarrow \infty$ ) as

$$
S[A] \sim S_{0}[A]+\sum_{n>0} \Lambda^{-n} S_{n}[A]
$$

where $S_{0}$ is independent of $\Lambda$. Recall from asymptotic analysis that this means that there exists a constant $C>0$ and $\Lambda_{0}>0$ such that for all $\Lambda>\Lambda_{0}$

$$
\left|S[A]-\sum_{n=0}^{N} \Lambda^{-n} S_{n}[A]\right| \leq C \Lambda^{-N-1}
$$

We interpret this in the following way:

- $S_{0}[A]$ is the physical action of interest, in this case the Yang-Mills action,

- $\sum_{n>0} \Lambda^{-n} S_{n}[A]$ is a higher-derivative (HD) gauge invariant action that acts as a regulator for $S_{0}[A]$ as in $[15,16]$ (cf. also [11, Section 4.4]); it vanishes as $\Lambda \rightarrow \infty$.

We consider $\Lambda$ as a regularizing parameter for, in this case, Yang-Mills theory. Eventually, after a perturbative quantization and renormalization, one sends $\Lambda$ to infinity. This motivates the fact that we start with the asymptotic expansion $\sum_{n \geq 0} \Lambda^{-n} S_{n}[A]$, rather than $S[A]$ as [13] do. It is illustrative to compare this with lattice gauge theory where a similar approach is taken: applying a lattice regularization to the Yang-Mills action one obtains the Wilson action. One then proceeds to quantize the latter, to eventually let the lattice spacing go to zero to recover a quantization of the original theory. In our case, we turn the spectral action in a higher-derivative action that regularizes Yang-Mills theory and reduces to the Yang-Mills action as the regularizing parameter $\Lambda$ goes to infinity.

Note that this is in contrast with the interpretation of $\Lambda$ as a large (but finite) physial scale, with the spectral action considered as an effective action, as in the original [4]. The different approach we take here has led to some controversy in the literature on the spectral action, with seemingly contradictory claims in [18] and [13]. However, as said, in the latter paper the authors consider the spectral action as given in Equation (1.1), without asymptotically expanding it in large $\Lambda$. They do not interpret $\Lambda$ as a regularizing parameter and of the action as a local HD gauge theory which at lowest order (in the derivatives) is the Yang-Mills action. Even though both started from the same spectral action principle, there is no contradiction between our results since one 
is really considering different Lagrangian theories. Here, the use in [13] of the word weak-field is misleading: the authors claim that the above asymptotic expansion (1.2) is only valid in the weak-field approximation. As a matter of fact, the estimates for the asymptotic expansion (cf. Eq. (1.3)) do not impose any bound on $S_{n}[A]$, nor on the fields $A$ and their momenta. Only the (invalid) consideration of the asymptotic expansion as a convergent series expansion might require such bounds.

\section{Higher-derivative Yang-Mills theory from the spectral action}

The main ingredient in the spectral action (1.1) is the Dirac operator on a compact Riemannian spin manifold $M$ with coefficients in a $S U(N)$-vector bundle equipped with a connection $A$. That is, locally we have

$$
D_{A}=i \gamma^{\mu}\left(\nabla_{\mu}+A_{\mu}\right)
$$

with $\nabla_{\mu}$ the spin connection and $A_{\mu}$ a skew-hermitian traceless matrix. The (hermitian) Dirac gamma matrices satisfy $\left\{\gamma_{\mu}, \gamma_{v}\right\}=2 g_{\mu \nu}$ and are represented on spinor space $S_{x}$ for each $x \in M$. The Dirac operator then acts as a self-adjoint operator in the Hilbert space $\mathscr{H}$ of $M_{N}(\mathbb{C})$-valued spinors:

$$
\mathscr{H}:=L^{2}(M, S) \otimes M_{N}(\mathbb{C}) .
$$

For simplicity, we take $M$ to be flat (i.e. vanishing Riemann curvature tensor) and 4-dimensional. Furthermore, we will assume that $f$ is a Laplace-Stieltjes transform:

$$
f(x)=\int_{t>0} e^{-t x^{2}} d \mu(t) .
$$

Proposition 1 ([5]). In the above notation, there is an asymptotic expansion (as $\Lambda \rightarrow \infty)$ :

$$
S[A] \sim \sum_{m>0} \Lambda^{4-m} f_{4-m} \int_{M} a_{m}\left(x, D_{A}^{2}\right),
$$

in terms of the Seeley-De Witt invariants of $D_{A}^{2}$. The coefficients are defined by $f_{k}:=\int t^{-k / 2} d \mu(t)$; in particular $f_{0}=f(0)$.

We can also compute the other coefficients $f_{k}$ explicitly:

Lemma 2 ([17]). The constants $f_{k}:=\int t^{-k / 2} d \mu(t)(k \in \mathbb{Z})$ are given by

1. $k>0: f_{k}=\frac{2}{\Gamma\left(\frac{k}{2}\right)} M_{k-1}[f]$ with $M_{k-1}$ the $k-1$ 'th moment of $f$,

2. $k \geq 0: f_{-2 k}=\frac{(-1)^{k} f^{(2 k)}(0)}{(2 k-1) ! !}$.

We will denote the asymptotic expansion at the right-hand side of Equation (2.1) by $S^{\Lambda}[A]$. Recall that the Seeley-De Witt coefficients $a_{m}\left(x, D_{A}^{2}\right)$ are gauge invariant (and coordinate independent) polynomials in the fields $A_{\mu}$. Indeed, the Weitzenböck formula gives

$$
D_{A}^{2}=-\left(\partial_{\mu}+A_{\mu}\right)\left(\partial^{\mu}+A^{\mu}\right)-\frac{1}{2} \gamma^{\mu} \gamma^{v} F_{\mu \nu}
$$


in terms of the curvature $F_{\mu \nu}=\partial_{\mu} A_{v}-\partial_{v} A_{\mu}+\left[A_{\mu}, A_{v}\right]$ of $A_{\mu}$. Consequently, a Theorem by Gilkey [12, Theorem 4.8.16] shows that (in this case) $a_{m}$ are polynomial gauge invariants in $F_{\mu \nu}$ and its covariant derivatives. The order ord of $a_{m}$ is $m$, where we set on generators:

$$
\operatorname{ord} A_{\mu_{1} ; \mu_{2} \cdots \mu_{k}}=k \text {. }
$$

Consequently, the curvature $F_{\mu \nu}$ has order 2 , and $F_{\mu_{1} \mu_{2} ; \mu_{3} \cdots \mu_{k}}$ has order $k$. For example, $a_{4}\left(x, D_{A}^{2}\right)$ is proportional to $\operatorname{Tr} F_{\mu v} F^{\mu v}$. In fact, we have an explicit expression for $S^{\Lambda}$ up to order $\Lambda^{-4}$.

Theorem 3. The action $S_{\leq 4}^{\Lambda}[A]:=f_{0} a_{4}\left(D_{A}^{2}\right)+\Lambda^{-2} f_{-2} a_{6}\left(D_{A}^{2}\right)+\Lambda^{-4} f_{-4} a_{8}\left(D_{A}^{2}\right)$ equals

$$
\begin{aligned}
S_{\leq 4}^{\Lambda}[A]= & -\frac{f_{0}}{8 \pi^{2}} \int \operatorname{Tr}_{N}\left(\frac{1}{3} F_{\mu v} F^{\mu v}\right)+\frac{f_{-2} \Lambda^{-2}}{8 \pi^{2}} \int \operatorname{Tr}_{N}\left(\frac{2}{15} F_{; \mu}^{\mu v} F_{; \rho}^{\rho v}+\frac{1}{45} F_{\mu}{ }^{v} F_{v}{ }^{\rho} F_{\rho}{ }^{\mu}\right) \\
& +\frac{f_{-4} \Lambda^{-4}}{8 \pi^{2}} \int \operatorname{Tr}_{N}\left(\frac{19}{1260} F_{\mu v} F^{\mu v} F_{\rho \sigma} F^{\rho \sigma}-\frac{13}{210} F_{\mu \nu} F^{v \rho} F^{\mu \sigma} F_{\sigma \rho}-\frac{1}{63} F_{\mu}{ }^{v} F_{v}{ }^{\rho} F_{\rho}{ }^{\sigma} F_{\sigma}{ }^{\mu}\right. \\
& \left.+\frac{4}{315} F_{\mu v} F_{\rho \sigma} F^{\mu v} F^{\rho \sigma}+\frac{4}{315} F_{; \sigma \mu}^{\sigma v} F_{v \rho} F^{\rho \mu}-\frac{17}{315} F_{; \rho}^{\rho \mu} F_{; \sigma}^{\sigma v} F_{\mu v}-\frac{1}{70} F_{; \rho \mu}^{\rho v} F_{\sigma v} ; \sigma \mu\right) .
\end{aligned}
$$

Proof. The Weitzenböck formula gives in the case of a flat manifold:

$$
D_{A}^{2}=-\frac{1}{2}\left\{\gamma^{\mu}, \gamma^{v}\right\} \nabla_{\mu} \nabla_{v}-\frac{1}{2}\left[\gamma^{\mu}, \gamma^{v}\right] \nabla_{\mu} \nabla_{v}=-\nabla_{\mu} \nabla^{\mu}-\frac{1}{2} \gamma^{\mu} \gamma^{v} F_{\mu v} \equiv \Delta-E
$$

The first three non-trivial heat coefficients of $e^{-t D_{A}^{2}}$ are on a flat manifold, and in a minimal basis $[19,1,2]$ given by

$$
\begin{aligned}
& a_{4}\left(D_{A}^{2}\right)=\frac{1}{(4 \pi)^{2}} \frac{1}{2} \int \operatorname{Tr}\left(E^{2}+\frac{1}{6} F_{\mu v} F^{\mu v}\right), \\
& a_{6}\left(D_{A}^{2}\right)=\frac{1}{(4 \pi)^{2}} \frac{1}{6} \int \operatorname{Tr}\left\{\frac{1}{2} E E_{; \mu}{ }^{\mu}+E^{3}-\frac{1}{10} F_{\mu \nu}{ }^{; \mu} F_{\rho v} ; \rho+\frac{1}{15} F_{\mu}{ }^{v} F_{v}{ }^{\rho} F_{\rho}{ }^{\mu}\right\} \text {, } \\
& a_{8}\left(D_{A}^{2}\right)=\frac{1}{(4 \pi)^{2}} \frac{1}{24} \int \operatorname{Tr}\left\{E^{4}+E^{2} E_{; \mu}{ }^{\mu}+\frac{4}{5} E^{2} F_{\mu \nu} F^{\mu v}+\frac{1}{5} E F_{\mu v} E F^{\mu v}-\frac{2}{5} E E_{; \mu} F^{v \mu}{ }_{; \nu}+\frac{1}{5}\left(E_{; \mu}{ }^{\mu}\right)^{2}\right. \\
& +\frac{17}{210} F_{\mu \nu} F^{\mu v} F_{\rho \sigma} F^{\rho \sigma}+\frac{2}{35} F_{\mu \nu} F^{v \rho} F^{\mu \sigma} F_{\sigma \rho}+\frac{1}{105} F_{\mu}{ }^{v} F_{v}{ }^{\rho} F_{\rho}{ }^{\sigma} F_{\sigma}{ }^{\mu}+\frac{1}{420} F_{\mu v} F_{\rho \sigma} F^{\mu v} F^{\rho \sigma} \\
& \left.+\frac{16}{105}\left(F_{; \sigma \mu}^{\sigma v} F_{v \rho} F^{\rho \mu}+F_{; \rho}^{\rho \mu} F_{; \sigma}^{\sigma v} F_{\mu \nu}\right)+\frac{1}{35} F_{; \rho \mu}^{\rho v} F_{\sigma \nu} ; \sigma \mu\right\}
\end{aligned}
$$

where we also used that the spinorial trace of $E=\frac{1}{2} \gamma^{\mu} \gamma^{v} F_{\mu \nu}$ vanishes.

Before substituting $E=\frac{1}{2} \gamma^{\mu} \gamma^{v} F_{\mu \nu}$ in the above expressions for $a_{4}, a_{6}$ and $a_{8}$, one can show the following crucial gamma matrix identities, when contracted with $F_{\mu v}$ 's and their covariant derivatives:

$$
\begin{aligned}
& \operatorname{Tr} E_{; \kappa_{1}} \lambda_{1} \ldots E_{; \kappa_{2}} \lambda_{2} \ldots=-2 \operatorname{Tr}_{N} F_{\mu v ; \kappa_{1}} \lambda_{1} \ldots F_{; \kappa_{2}}^{\mu v} \lambda_{2} \ldots \\
& \operatorname{Tr} E_{; \kappa_{1} \lambda_{1} \ldots E_{; \kappa_{2}} \lambda_{2} \ldots E_{; \kappa_{3}} \lambda_{3} \ldots}=4 \operatorname{Tr}_{N} F_{\mu}{ }_{; \kappa_{1} \lambda_{1} \ldots} F_{V}{ }^{\rho}{ }_{; \kappa_{2}} \lambda_{2} \ldots F_{\rho}{ }_{; \kappa_{3} \lambda_{3} \ldots}{ }^{\mu}
\end{aligned}
$$

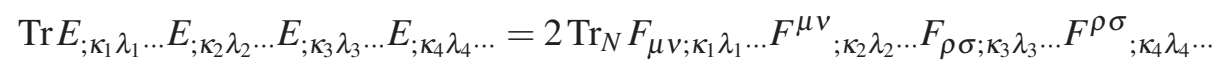

$$
\begin{aligned}
& -8 \operatorname{Tr}_{N} F_{\mu v ; \kappa_{1}} \lambda_{1} \ldots F_{; \kappa_{2} \lambda_{2} \ldots F^{\mu \sigma}}^{\mu \sigma}{ }_{; \kappa_{3}} \lambda_{3} \ldots F_{\sigma \rho ; \kappa_{4}} \lambda_{4} \ldots
\end{aligned}
$$

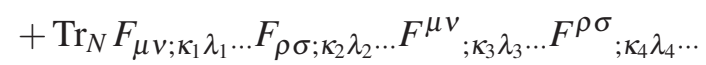

$$
\begin{aligned}
& +4 \operatorname{Tr}_{N} F_{\mu}^{v}{ }_{; \kappa_{1}} \lambda_{1} \ldots F_{v}{ }^{\rho} ; \kappa_{2} \lambda_{2} \ldots F_{\rho}{ }_{; \kappa_{3} \lambda_{3} \ldots} F_{\sigma}^{\mu}{ }_{; \kappa_{4}} \lambda_{4} \ldots
\end{aligned}
$$


where the $\kappa_{i}, \lambda_{i}, \cdots$ indicate an arbitrary number of covariant derivatives.

Let us start with $a_{4}$. Then using Eq. (2.3) we find

$$
\begin{aligned}
\operatorname{Tr} E^{2} & =-2 \operatorname{Tr}_{N} F_{\mu v} F^{\mu v}, \\
\operatorname{Tr} F_{\mu v} F^{\mu v} & =4 \operatorname{Tr}_{N} F_{\mu v} F^{\mu v} .
\end{aligned}
$$

The coefficient in front of $\int \operatorname{Tr}_{N} F_{\mu v} F^{\mu v}$ in $a_{4}$ thus becomes

$$
\frac{1}{(4 \pi)^{2}} \frac{1}{2}\left(-2+4 \times \frac{1}{6}\right)=\frac{1}{8 \pi^{2}}\left(-\frac{1}{3}\right)
$$

where the 4 on the left-hand side is due to the trace in spinor space.

Next, for $a_{6}$ we derive from Eq. (2.3) and (2.4) for the first two terms that

$$
\int \operatorname{Tr}\left\{\frac{1}{2} E E_{; \rho}{ }^{\rho}+E^{3}\right\}=\int\left(-\operatorname{Tr}_{N} F_{\mu v} F_{\rho}^{\mu v ; \rho}+4 \operatorname{Tr}_{N} F_{\mu}{ }^{v} F_{v}{ }^{\rho} F_{\rho}{ }^{\mu}\right)=\int 2 \operatorname{Tr}_{N} F_{\mu \nu} ; \mu F_{; \rho}^{\rho v}
$$

where we have used in addition the relation ${ }^{1}$

$$
\int \operatorname{Tr}_{N} F_{\mu v} F_{\rho}^{\mu v ; \rho}=\int\left(-2 \operatorname{Tr}_{N} F_{\mu v} ; \mu F_{; \rho}^{\rho v}+4 \operatorname{Tr}_{N} F_{\mu}{ }^{v} F_{v}{ }^{\rho} F_{\rho}{ }^{\mu}\right)
$$

which follows from partial integration and the Bianchi identity. The coefficient in front of $\int \operatorname{Tr}_{N} F_{\mu v} ; \mu F_{; \rho}^{\rho v}$ in $a_{6}$ thus becomes

$$
\frac{1}{(4 \pi)^{2}} \frac{1}{6}\left(2-4 \times \frac{1}{10}\right)=\frac{1}{8 \pi^{2}} \frac{2}{15}
$$

We now turn to the more involved coefficient $a_{8}$. We first determine the first 6 terms in $a_{8}$, depending on $E$ and its derivatives. Using (2.5) we find

$$
\begin{aligned}
\operatorname{Tr} E^{4}= & 2 \operatorname{Tr}_{N} F_{\mu v} F^{\mu v} F_{\rho \sigma} F^{\rho \sigma}-8 \operatorname{Tr}_{N} F_{\mu v} F^{v \rho} F^{\mu \sigma} F_{\sigma \rho} \\
& +4 \operatorname{Tr}_{N} F_{\mu}{ }^{v} F_{v}{ }^{\rho} F_{\rho}{ }^{\sigma} F_{\sigma}{ }^{\mu}+\operatorname{Tr}_{N} F_{\mu v} F_{\rho \sigma} F^{\mu v} F^{\rho \sigma}
\end{aligned}
$$

For the cubic term in $E$ we apply (2.4), partial integration and the Bianchi identity to obtain

$$
\int \operatorname{Tr} E^{2} E_{; \sigma}{ }^{\sigma}=\int\left(8 \operatorname{Tr}_{N} F_{\mu \nu} F^{v \rho} F^{\mu \sigma} F_{\sigma \rho}-8 \operatorname{Tr}_{N} F_{\mu}{ }^{v} F_{v}{ }^{\rho} F_{\rho}{ }^{\sigma} F_{\sigma}^{\mu}+8 \operatorname{Tr}_{N} F^{\sigma v} ; \sigma \mu F_{v \rho} F^{\rho \mu}\right) .
$$

For the terms quadratic in $E$ we apply (2.3), partial integration and the Bianchi identity to obtain the subsequent identities

$$
\begin{aligned}
\int \frac{4}{5} \operatorname{Tr} E^{2} F_{\mu v} F^{\mu v} & =\int-\frac{8}{5} \operatorname{Tr}_{N} F_{\mu v} F^{\mu v} F_{\rho \sigma} F^{\rho \sigma} \\
\int \frac{1}{5} \operatorname{Tr} E F_{\mu v} E F^{\mu v} & =\int-\frac{2}{5} \operatorname{Tr}_{N} F_{\mu v} F_{\rho \sigma} F^{\mu v} F^{\rho \sigma} \\
\int-\frac{2}{5} \operatorname{Tr} E E_{; \mu} F_{; \nu}^{v \mu} & =\int\left(-\frac{8}{5} \operatorname{Tr}_{N} F_{; \sigma}^{\sigma \rho} F_{; \mu}^{\mu v} F_{\rho v}-\frac{8}{5} \operatorname{Tr}_{N} F_{; \sigma \mu}^{\sigma \rho} F_{\rho v} F^{v \mu}\right)
\end{aligned}
$$

\footnotetext{
${ }^{1}$ Here we correct for a typo at the end of the proof of Proposition 27 in [17].
} 
and finally

$$
\begin{aligned}
& \int \frac{1}{5} \operatorname{Tr}\left(E_{; \mu}{ }^{\mu}\right)^{2}=\int\left(-\frac{16}{5} \operatorname{Tr}_{N} F_{\mu v} F^{v \rho} F^{\mu \sigma} F_{\sigma \rho}+\frac{16}{5} \operatorname{Tr}_{N} F_{\mu}{ }^{v} F_{v}{ }^{\rho} F_{\rho}{ }^{\sigma} F_{\sigma}{ }^{\mu}\right. \\
& \left.-\frac{32}{5} \operatorname{Tr}_{N} F_{; \rho v}^{\rho \mu} F_{\mu \sigma} F^{\sigma v}-\frac{4}{5} \operatorname{Tr}_{N} F_{\sigma v} ; \sigma \mu F_{; \rho \mu}^{\rho v}-\frac{8}{5} \operatorname{Tr}_{N} F_{; \rho}^{\rho \mu} F_{; \sigma}^{\sigma v} F_{\mu v}\right)
\end{aligned}
$$

The only thing that remains to be done is to collect all the terms and compute their coefficients:

$$
\begin{aligned}
& \int \operatorname{Tr}_{N} F_{\mu v} F^{\mu v} F_{\rho \sigma} F^{\rho \sigma}: \frac{1}{(4 \pi)^{2}} \frac{1}{24}\left(2-\frac{8}{5}+4 \times \frac{17}{210}\right)=\frac{1}{8 \pi^{2}} \frac{19}{1260} \\
& \int \operatorname{Tr}_{N} F_{\mu v} F^{v \rho} F^{\mu \sigma} F_{\sigma \rho}: \frac{1}{(4 \pi)^{2}} \frac{1}{24}\left(-8+8-\frac{16}{5}+4 \times \frac{2}{35}\right)=\frac{1}{8 \pi^{2}}\left(-\frac{13}{210}\right) \\
& \int \operatorname{Tr}_{N} F_{\mu}^{v} F_{v}{ }^{\rho} F_{\rho}{ }^{\sigma} F_{\sigma}{ }^{\mu}: \frac{1}{(4 \pi)^{2}} \frac{1}{24}\left(4-8+\frac{16}{5}+4 \times \frac{1}{105}\right)=\frac{1}{8 \pi^{2}}\left(-\frac{1}{63}\right) \\
& \int \operatorname{Tr}_{N} F_{\mu v} F_{\rho \sigma} F^{\mu v} F^{\rho \sigma}: \frac{1}{(4 \pi)^{2}} \frac{1}{24}\left(1-\frac{2}{5}+4 \times \frac{1}{420}\right)=\frac{1}{8 \pi^{2}} \frac{4}{315} \\
& \int \operatorname{Tr}_{N} F_{; \sigma \mu}^{\sigma v} F_{v \rho} F^{\rho \mu}: \frac{1}{(4 \pi)^{2}} \frac{1}{24}\left(8-\frac{8}{5}-\frac{32}{5}+4 \times \frac{16}{105}\right)=\frac{1}{8 \pi^{2}} \frac{4}{315} \\
& \int \operatorname{Tr}_{N} F_{; \rho}^{\rho \mu} F_{; \sigma}^{\sigma v} F_{\mu v}: \frac{1}{(4 \pi)^{2}} \frac{1}{24}\left(-\frac{8}{5}-\frac{8}{5}+4 \times \frac{16}{105}\right)=\frac{1}{8 \pi^{2}}\left(-\frac{17}{315}\right) \\
& \int \operatorname{Tr}_{N} F_{; \rho \mu}^{\rho v} F_{\sigma v} ; \sigma \mu: \frac{1}{(4 \pi)^{2}} \frac{1}{24}\left(-\frac{4}{5}+4 \times \frac{1}{35}\right)=\frac{1}{8 \pi^{2}}\left(-\frac{1}{70}\right)
\end{aligned}
$$

The appearance of the Yang-Mills action is the main motivation to study this model. As explained in the introduction, we consider the terms in $S^{\Lambda}[A]$ proportional to $\Lambda^{-2}$ and $\Lambda^{-4}$ as regulators for the Yang-Mills action. Let us first focus on the free quadratic part of the action $S^{\Lambda}[A]$.

Theorem 4 ([17]). There is the following asymptotic expansion $($ as $\Lambda \rightarrow \infty)$ for the free part of the spectral action on a flat background manifold $M$

$$
S_{\text {free }}[A] \sim-\int \operatorname{Tr} \hat{F}_{\mu \nu} \varphi_{\Lambda}(\Delta)\left(\hat{F}^{\mu v}\right)
$$

where

$$
\varphi_{\Lambda}(\Delta)=\sum_{k \geq 0}(-1)^{k} \Lambda^{-2 k} f_{-2 k} c_{k} \Delta^{k}, \quad c_{k}=\frac{1}{8 \pi^{2}} \frac{(k+1) !}{(2 k+3)(2 k+1) !},
$$

is an expansion in the Laplacian $\Delta$ and $\hat{F}_{\mu \nu}=\partial_{\mu} A_{\nu}-\partial_{v} A_{\mu}$.

Remark 5. Even though the above expansion for $S_{\text {free }}[A]$ is asymptotic for large $\Lambda$, it is interesting to consider the corresponding actual sum that defines $\varphi_{\Lambda}$. In particular, this allows to confront our results once again with [13], by considering the large momentum limit of the full sum. Thus, consider

$$
\varphi_{\Lambda}(x)=\frac{1}{8 \pi^{2}} \int_{t \geq 0} \sum_{k=0}^{\infty} \frac{(k+1) !}{(2 k+3)(2 k+1) !}\left(-t x / \Lambda^{2}\right)^{k} d \mu(t) .
$$


One finds that

$$
\varphi_{\Lambda}(x)=\frac{1}{8 \pi^{2}} \int\left(\left(\frac{\Lambda}{\sqrt{t x}}+2 \frac{\Lambda^{3}}{(t x)^{3 / 2}}\right) F\left(\frac{\sqrt{t x}}{2 \Lambda}\right)-\frac{\Lambda^{2}}{t x}\right) d \mu(t)
$$

Here, the Dawson function $F$ is defined in terms of the error function by

$$
F(z)=\frac{\sqrt{\pi}}{2} e^{-z^{2}} \operatorname{erfi}(z)
$$

The asymptotic behaviour of $\varphi_{\Lambda}\left(p^{2}\right)$ as $p^{2} \rightarrow \infty$ can then be determined to be

$$
\varphi_{\Lambda}\left(p^{2}\right) \underset{p^{2} \rightarrow \infty}{\sim} \frac{1}{2 \pi^{2}} f_{4} \Lambda^{4} p^{-4}+\cdots
$$

using that $F(z) \sim 1 / 2 z+1 / 4 z^{3}+\cdots$ as $z \rightarrow \infty$. It is striking that already at this heuristic level it would lead to the same conclusion on the UV-behaviour of the spectral action as in [13]. For more details on this comparison, we refer to [17].

In our case, being interested in the action $S_{\leq 4}^{\Lambda}[A]$ up to order $\Lambda^{-4}$, we put $f_{-6}=f_{-8}=\cdots=0$ in the above expansion for $\varphi_{\Lambda}$. Thus, $\varphi_{\Lambda}$ is the following polynomial in $\Delta$ :

$$
\varphi_{\Lambda}(\Delta)=f_{0} c_{0}-f_{-2} \Lambda^{-2} c_{2} \Delta^{2}+f_{-4} \Lambda^{-4} c_{4} \Delta^{4}=\frac{1}{8 \pi^{2}}\left(\frac{f(0)}{3}+\frac{f^{(2)}(0)}{15} \Delta / \Lambda^{2}+\frac{f^{(4)}(0)}{420}\left(\Delta / \Lambda^{2}\right)^{2}\right)
$$

where for the second equality we have used Lemma 2.

Moreover, the free part of $S_{\leq 4}^{\Lambda}$ is given by the following polynomial expression:

$$
S_{\text {free }, \leq 4}^{\Lambda}[A]=-\int \operatorname{Tr} \hat{F}_{\mu v} \varphi_{\Lambda}(\Delta)\left(\hat{F}^{\mu v}\right)
$$

This form motivates the interpretation of $S_{\text {free }}^{\Lambda}[A]$ (and of $S^{\Lambda}[A]$ ) as a higher-derivative gauge theory. As we will see below, this indeed regularizes the theory in such a way that $S^{\Lambda}[A]$ defines a superrenormalizable field theory.

Remark 6. The above form of $\varphi_{\Lambda}$ can be confronted with the free part of the action that was computed in Theorem 3. From there, we find

$$
S_{\leq 4}=\frac{1}{8 \pi^{2}} \int\left(-\frac{f_{0}}{3} \operatorname{Tr}_{N} \hat{F}_{\mu v} \hat{F}^{\mu v}+\frac{f_{-2}}{15} \operatorname{Tr}_{N} \hat{F}_{\mu v} \Delta\left(\hat{F}^{\mu v}\right)-\frac{f_{-4}}{140} \operatorname{Tr}_{N} \hat{F}_{\mu v} \Delta^{2}\left(\hat{F}^{\mu v}\right)\right)+\mathscr{O}\left(A^{3}\right)
$$

using also that $\operatorname{Tr}_{N} F_{\mu v ; \rho}{ }^{\rho} F^{\mu v} ; \sigma=2 \operatorname{Tr}_{N} F_{\rho \mu}{ }^{\rho v}{ }^{\rho} F^{\sigma \mu}{ }_{; \sigma v}+\mathscr{O}\left(A^{3}\right)$. This is in concordance with the above expression for $S_{\text {free }, \leq 4}[A]$ since $c_{0}=1 / 24 \pi^{2}, c_{1}=1 / 120 \pi^{2}$ and $c_{2}=1 / 1120 \pi^{2}$ as appearing in Theorem 4.

In the following, we will drop the subscript $\leq 4$ and assume $f_{-6}=f_{-8}=\cdots=0$. 


\section{Higher-derivative gauge fixing in the YM-system}

As usual for a gauge theory, the quadratic form defined by $S_{\text {free }}[A]$ is not invertible: we need to fix the gauge.

First, we introduce $\mathfrak{s u}(N)$-valued ghost fields $\bar{C}, C$ and an auxiliary field $h$, which is also $\mathfrak{s u}(N)$ valued. These fields are transformed into eachother by the BRST-differential $s$, which is defined by

$$
s A_{\mu}=\partial_{\mu} C+\left[A_{\mu}, C\right] ; \quad s C=-\frac{1}{2}[C, C] ; \quad s \bar{C}=-h, \quad s h=0 .
$$

Note that these are the usual formulas for the BRST-differential, so that $s^{2}=0$. The gauge-fixing fermion is given by

$$
\Psi^{\Lambda}=-\int \operatorname{Tr}_{N} \varphi_{\Lambda}(\Delta)(\bar{C})\left(\frac{1}{2} \xi h+\partial_{\mu} A^{\mu}\right) .
$$

so that gauge-fixing is achieved by adding $s\left(\Psi^{\Lambda}\right)$ to the asymptotically expanded spectral action. It is then automatic that

Proposition 7. The sum $S^{\Lambda}[A]+s\left(\Psi^{\Lambda}\right)$ is BRST-invariant: $s\left(S^{\Lambda}[A]+s\left(\Psi^{\Lambda}\right)\right)=0$.

In order to derive the gauge propagator, we need to invert the quadratic forms in $A^{\mu}$ and $C$ given by $S_{\text {free }}^{\Lambda}[A]+s\left(\Psi^{\Lambda}\right)$, after solving the equations of motion for the auxiliary field $h$. This is only possible if $\varphi_{\Lambda}\left(p^{2}\right)$ is nonvanishing, in which case the propagator is given by

$$
D_{\mu v}^{a b}(p ; \Lambda)=\left[g_{\mu v}-(1-\xi) \frac{p_{\mu} p_{v}}{p^{2}}\right] \frac{\delta^{a b}}{p^{2} \varphi_{\Lambda}\left(p^{2}\right)} .
$$

The non-vanishing of $\varphi_{\Lambda}\left(p^{2}\right)$ can be guaranteed by assuming that $f^{(2)}(0), f^{(4)}(0) \geq 0$, which is what we do in the following. The behaviour of $D_{\mu \nu}^{a b}$ for large $p$ will be discussed in more detail in the next subsection.

Similarly, we derive that the propagator for the Faddeev-Popov ghost fields and their propagator is

$$
\widetilde{D}^{a b}(p ; \Lambda)=\frac{\delta^{a b}}{p^{2} \varphi_{\Lambda}\left(p^{2}\right)} .
$$

referring to [17] for more details.

\subsection{Renormalization of the asymptotically expanded YM-spectral action}

We now discuss the UV-behaviour of the field theory defined by $S^{\Lambda}[A]$, still keeping $f_{-6}=$ $f_{-8}=\cdots=0$. First, we easily derive from the polynomial structure of $\varphi_{\Lambda}\left(p^{2}\right)$ that the propagators of both the gauge field and the ghost field behave as $|p|^{-6}$ as $|p| \rightarrow \infty$. Indeed, in this case:

$$
\varphi_{\Lambda}\left(p^{2}\right)=\frac{1}{8 \pi^{2}}\left(\frac{f(0)}{3}+\frac{f^{(2)}(0)}{15} p^{2} / \Lambda^{2}+\frac{f^{(4)}(0)}{420}\left(p^{2} / \Lambda^{2}\right)^{2}\right)
$$

Moreover, the maximum weights of the interaction in terms of powers of momenta is given by $8-i$, where $i$ is the valence of the vertex. We will use $v_{k}$ to indicate the number of gauge interaction vertices of valence $k$, and with $\widetilde{v}$ the number of ghost-gauge vertices. 
Let us now find an expression for the superficial degree of divergence $\omega$ of a Feynman graph consisting of $I$ internal gauge edges, $\widetilde{I}$ internal ghost edges, $v_{k}$ valence $k$ gauge vertices and $\widetilde{v}$ ghost-gauge vertices. In 4 dimensions, we find at loop order $L$ :

$$
\omega \leq 4 L-6 I-6 \widetilde{I}+\sum_{i=3}^{8} v_{i}(8-i)+5 \widetilde{v}
$$

Lemma 8. Let $E$ and $\widetilde{E}$ denote the number of external gauge and ghost edges, respectively. The superficial degree of divergence of the graph satisfies:

$$
\omega \leq-4(L-1)+4-(E+\widetilde{E}) .
$$

Proof. We use the relations

$$
2 I+E=\sum_{i} i v_{i}+\widetilde{v} ; \quad 2 \widetilde{I}+\widetilde{E}=2 \widetilde{v}
$$

where $E$ and $\widetilde{E}$ are the number of external gauge and ghost legs, respectively. Indeed, these formulas count the number of half (gauge/ghost) edges in a graph in two ways: from the number of edges and from the valences of the vertices. We use them to substitute for $2 I$ and $2 \widetilde{I}$ in the above expression for $\omega$ so as to obtain

$$
\omega \leq 4 L-8 I-8 \widetilde{I}+8\left(\sum_{i} v_{i}+\widetilde{v}\right)-(E+\widetilde{E})
$$

from which the result follows at once from Euler's formula $L=I+\widetilde{I}-\sum_{i} v_{i}-\widetilde{v}+1$.

We conclude that the theory defined by $S^{\Lambda}[A]$ is superrenormalizable. Indeed, $\omega<0$ if $L \geq 2$ : all Feynman graphs are finite at loop order greater than 1 . If $L=1$, then there are finitely many graphs which are divergent, namely those for which $E+\widetilde{E} \leq 4$. We conclude that the asymptotically expanded spectral action for the Yang-Mills system is superrenormalizable.

Of course, the spectral action being a gauge theory, there is more to renormalizability than just power counting: we have to establish gauge invariance of the counterterms. We already know that the counterterms needed to render the perturbative quantization of $S^{\Lambda}[A]$ finite are of order 4 or less in the fields and arise only from one-loop graphs. The key property of the effective action at one loop is that it is supposed to be BRST-invariant:

$$
s\left(\Gamma_{1}\right)=0 .
$$

In particular, assuming a regularization compatible with gauge invariance, the divergent part $\Gamma_{1, \infty}$ is BRST-invariant. Results from [7, 8, 9, 3, 10] on BRST-cohomology for Yang-Mills type theories ascertain that the only BRST-closed functional of order 4 or less in the fields is represented by

$$
\delta Z \int F_{\mu v} F^{\mu v}
$$

for some constant $\delta Z$. 
Theorem 9. The action $S^{\Lambda}[A]$ for the Yang-Mills system on a flat background manifold is renormalizable. The renormalized (asymptotically expanded) spectral action $S_{\mathrm{ren}}^{\Lambda}[A]$ is obtained from $S^{\Lambda}[A]$ by the following redefinition of the coefficient $f_{0}$ :

$$
f_{0} \mapsto f_{0}+24 \pi^{2} \delta Z
$$

leaving all other coefficients $f_{-2 k}(k \neq 0)$ invariant.

Intriguingly, renormalization of $S^{\Lambda}[A]$ can thus be accomplished merely by shifting the function $f$ by a constant amount $24 \pi^{2} \delta Z$. If we adopt dimensional-regularization and minimal subtraction to renormalize the one-loop diagrams, then the counterterms are typically of the following form

$$
\delta Z=c\left(\frac{1}{z}+2 \ln \mu\right)
$$

for some constant $\mathrm{c}$, and in terms of the usual mass scale $\mu$. Defining a bare quantity $f_{0}^{B}$ by

$$
f_{0}^{B}:=f_{0}+24 \pi^{2} c\left(\frac{1}{z}+2 \ln \mu\right)
$$

its supposed independence of the mass scale $\mu$ implies that

$$
\mu \frac{\partial f_{0}}{\partial \mu}=-48 \pi^{2} c
$$

This defines the renormalization group flow on the spectral function $f$, which in this case is linear on the coefficient $f_{0}$. Also, since the only divergences appear at one loop, these equations are expected to hold at the non-perturbative level.

\section{Outlook}

The above linear beta-function seems to be at odds with the usual Yang-Mills beta-function. This is due to the fact that one would have to consider the divergences that appear in the Feynman amplitudes defined by $S^{\Lambda}$ as $\Lambda \rightarrow \infty$. As in [14], we expect that besides the above $1 / z$-divergences (in dim-reg), also terms will appear that are proportional to $\ln \Lambda, \Lambda^{2}$, etc. This is a consequence of the adopted hybrid regularization, combining higher-derivative and dimensional regularization. Once both types of divergences have been countered, the beta-functions should be determined and compared to the usual (dim-reg) Yang-Mills beta-function.

\section{References}

[1] I. Avramidi. A covariant technique for calculation of one loop effective action. Nucl. Phys. B355 (1991) 712-754.

[2] A. Bel'kov, A. Lanyov, and A. Schaale. Calculation of heat kernel coefficients and usage of computer algebra. Comput.Phys.Commun. 95 (1996) 123-130.

[3] F. Brandt, N. Dragon, and M. Kreuzer. Lie algebra cohomology. Nucl. Phys. B332 (1990) 250.

[4] A. H. Chamseddine and A. Connes. Universal formula for noncommutative geometry actions: Unifications of gravity and the standard model. Phys. Rev. Lett. 77 (1996) 4868-4871. 
[5] A. H. Chamseddine and A. Connes. The spectral action principle. Commun. Math. Phys. 186 (1997) $731-750$.

[6] A. H. Chamseddine and A. Connes. Noncommutative Geometry as a Framework for Unification of all Fundamental Interactions including Gravity. Part II. To appear.

[7] J. A. Dixon. Calculation of BRS cohomology with spectral sequences. Commun. Math. Phys. 139 (1991) 495-526.

[8] M. Dubois-Violette, M. Talon, and C. M. Viallet. BRS algebras: Analysis of the consistency equations in gauge theory. Commun. Math. Phys. 102 (1985) 105.

[9] M. Dubois-Violette, M. Talon, and C. M. Viallet. Results on BRS cohomology in gauge theory. Phys. Lett. B158 (1985) 231.

[10] M. Dubois-Violette, M. Henneaux, M. Talon, and C.-M. Viallet. Some results on local cohomologies in field theory. Phys. Lett. B267 (1991) 81-87.

[11] L. Faddeev and A. Slavnov. Gauge Fields. Introduction to Quantum Theory. Benjaming Cummings, 1980.

[12] P. B. Gilkey. Invariance theory, the heat equation, and the Atiyah-Singer index theorem, volume 11 of Mathematics Lecture Series. Publish or Perish Inc., Wilmington, DE, 1984.

[13] B. Iochum, C. Levy, and D. Vassilevich. Spectral action beyond the weak-field approximation. arXiv:1108.3749.

[14] C. Martin and F. Ruiz Ruiz. Higher covariant derivative regulators and nonmultiplicative renormalization. Phys. Lett. B343 (1995) 218-224.

[15] A. A. Slavnov. Invariant regularization of nonlinear chiral theories. Nucl. Phys. B31 (1971) 301-315.

[16] A. A. Slavnov. Ward identities in gauge theories. Theor. Math. Phys. 10 (1972) 99-107.

[17] W. D. van Suijlekom. Renormalization of the asymptotically expanded Yang-Mills spectral action. To appear in Commun. Math. Phys. [arXiv:1104.5199].

[18] W. D. van Suijlekom. Renormalization of the spectral action for the Yang-Mills system. JHEP 1103 (2011) 146.

[19] A. van de Ven. Explicit counter action algorithms in higher dimensions. Nucl. Phys. B250 (1985) 593. 